\title{
The faint supernova remnant G $116.5+1.1$ and the detection of a new candidate remnant
}

\author{
F. Mavromatakis ${ }^{1}$, P. Boumis ${ }^{2}$, E. Xilouris ${ }^{2}$, J. Papamastorakis ${ }^{1,3}$, and J. Alikakos ${ }^{2,4}$ \\ 1 University of Crete, Physics Department, PO Box 2208, 71003 Heraklion, Crete, Greece \\ e-mail: fotis@physics.uoc.gr \\ 2 Institute of Astronomy \& Astrophysics, National Observatory of Athens, I. Metaxa \& V. Pavlou, P. Penteli, 15236 Athens, \\ Greece \\ ${ }^{3}$ Foundation for Research and Technology-Hellas, PO Box 1527, 71110 Heraklion, Crete, Greece \\ 4 Astronomical Laboratory, Department of Physics, University of Patras, 26500 Rio-Patras, Greece
}

Received 15 October 2004 / Accepted 13 January 2005

\begin{abstract}
The extended supernova remnant G 116.5+1.1 was observed in the optical emission lines of $\mathrm{H} \alpha+[\mathrm{N}$ II], [S II] and [O III]; deep long slit spectra were also obtained. The morphology of the remnant's observed emission is mainly diffuse and patchy in contrast to the known filamentary emission seen along the western limb. The bulk of the detected emission in the region appears unrelated to the remnant but there is one area of emission in the south-east which is characterized by a [S II]/H $\alpha$ ratio of $\sim 0.5$, implying a possible relation to $\mathrm{G} 116.5+1.1$. If this is actually the case, it would imply a more extended remnant than previously realized. Emission in the [O III] $5007 \AA$ line image is not detected, excluding moderate or fast velocity shocks running into ionized interstellar clouds. Our current estimate of the distance to $\mathrm{G} 116.5+1.1$ of $\sim 3 \mathrm{kpc}$ is in agreement with earlier estimates and implies a very extended remnant $(69 \mathrm{pc} \times 45 \mathrm{pc})$.

Observations further to the north-east of G 116.5+1.1 revealed a network of filamentary structures prominent in $\mathrm{H} \alpha+[\mathrm{N}$ II] and $[\mathrm{S} \mathrm{II}]$ but failed to detect [O III] line emission. Long slit spectra in a number of positions provide strong evidence that this newly detected emission arises from shock heated gas. Typical $\mathrm{H} \alpha$ fluxes lie in the range of 9 to $17 \times 10^{-17} \mathrm{erg} \mathrm{s}^{-1} \mathrm{~cm}^{-2} \operatorname{arcsec}^{-2}$, while low electron densities are implied by the intensities of the sulfur lines. Weak emission from the medium ionization line at $5007 \AA$ is detected in only one spectrum. Cool dust emission at 60 and 100 microns may be correlated with the optical emission in a limited number of positions. Surpisingly, radio emission is not detected in published surveys suggesting that the new candidate remnant may belong to the class of "radio quiet" supernova remnants.
\end{abstract}

Key words. ISM: general - ISM: supernova remnants - ISM: individual objects: G 116.5+1.1

\section{Introduction}

Supernova remnants are important ingredients of a galaxy because of the huge amount of energy released during the initial explosion, while in subsequent phases heavy elements are mixed into the interstellar medium (ISM). Observations of supernova remnants in X-ray wavelengths allow us to directly probe the hot gas inside the primary shock wave. Optical observations offer an important tool for the study of the interaction of the shock wave with dense concentrations of gas found in the ISM. The detection and observation of well extended remnants is a difficult task since their surface brightness can be quite low but such observations would contribute significantly towards an unbiased supernova remnant catalog.

One such extended object is G 116.5+1.1 located in the Perseus Arm. Reich \& Braunsfurth (1981) used data at 2.7 and $1.4 \mathrm{GHz}$ to establish the non-thermal nature of the radio emission and classified $\mathrm{G} 116.5+1.1$ as a supernova remnant. Identifying certain HI features as related to the object, they obtained a distance of $4.4( \pm 0.4) \mathrm{kpc}$. Fich (1986) in a study of large-scale structures in the Perseus Arm suggested that $\mathrm{G} 116.5+1.1$ as well as CTB 1 are found at the edges of an HI supershell. Reich \& Braunsfurth (1981) provided quantitative estimates of the remnant's explosion energy, shock velocity, ISM density etc., but with significant uncertainties. Unfortunately, there have not been any dedicated X-ray observations, while no emission was detected in the ROSAT All-Sky survey.

Fesen et al. (1997) obtained $\mathrm{H} \alpha$ and [S II] images $\left(59^{\prime} \times\right.$ $52^{\prime}$ wide) in the north-west and detected a faint long $\left(\sim 30^{\prime}\right)$ filament. Low dispersion spectra at the position of the filament verified the shock heated nature of this optical emission. Because our knowledge of this remnant is limited to optical wavelengths we obtained the deepest currently available CCD images of the full field of $\mathrm{G} 116.5+1.1$ in the emission lines of $\mathrm{H} \alpha+[\mathrm{N} \mathrm{II}]$, [S II] and [O III]. The detection of new filamentary structures to the north-east of $\mathrm{G} 116.5+1.1$ led to additional imagery in the same filters. Deep long slit spectra were also acquired at 
Table 1. Imaging log.

\begin{tabular}{llll}
\hline \hline $\mathrm{H} \alpha+[\mathrm{N} \mathrm{II}]$ & {$[\mathrm{S} \mathrm{II}]$} & {$[\mathrm{O} \mathrm{III}]$} & Date \\
\hline $2400^{a}(1)^{b}$ & $4800(2)$ & - & $09-08-2002$ \\
$2400(1)$ & $2400(1)$ & - & $10-08-2002$ \\
- & - & $7200(3)$ & $11-08-2002$ \\
$2400(1)$ & $2400(1)$ & - & $12-08-2002$ \\
$4800(2)$ & $4800(2)$ & - & $13-08-2002$ \\
\hline $4800(2)^{*}$ & - & - & $14-06-2004$ \\
$4800(2)^{*}$ & - & - & $15-06-2004$ \\
- & $2400(1)$ & - & $20-06-2004$ \\
$2400(1)$ & - & - & $21-06-2004$ \\
$2400(1)$ & $2400(1)$ & $2400(1)$ & $22-06-2004$ \\
$6560^{c}\left(75^{d}\right)$ & $6708(20)$ & $5005(28)$ & \\
\hline
\end{tabular}

${ }^{a}$ Total exposure time in $\mathrm{s}$.

${ }^{b}$ Number of individual frames.

${ }^{c}$ Central wavelength in $\AA$.

${ }^{d}$ Full width at half maximum in $\AA$.

* Each of the two frames covers different fields.

various locations in order to study in more detail the new structures. Information about the observations and the data reduction is given in Sect. 2. In Sects. 3 and 4 the results of the imaging and spectral observations are presented, while in Sect. 5 we report on observations in wavelengths other than optical. In Sect. 6 we discuss the properties of $\mathrm{G} 116.5+1.1$ and the new candidate remnant. In Sect. 7 we summarize the results of this work.

\section{Observations}

\subsection{Optical images}

The imagery was performed with the $0.3 \mathrm{~m}$ wide-field Schmidt-Cassegrain telescope at the Skinakas Observatory, Crete, Greece. Multiple pointings were performed from August 9 to 13, 2002 in order to map the whole field of $\mathrm{G} 116.5+1.1$ as defined by its radio emission. The field to the east of $\mathrm{G} 116.5+1.1$ was observed on June 12, 14-15, and 20-22, 2004. In all cases, the telescope was equipped with a $1024 \times 1024$ Thomson CCD providing a $70^{\prime} \times 70^{\prime}$ field of view and an image scale of $4^{\prime \prime}$ per pixel. The filters isolating the $\mathrm{H} \alpha+[\mathrm{N}$ II],$[\mathrm{S} \mathrm{II}]$, and [O III] emission lines were used in these observations. Details about the filter characteristics and the exposure times can be found in Table 1. An astrometric solution was calculated for all image frames with the aid of the HST Guide star catalogue (Lasker et al. 1999) and all frames were subsequently projected to a common origin on the sky for further operations. The equatorial coordinates quoted in this work refer to epoch J2000.

The data were reduced using standard IRAF and MIDAS routines. The available frames were bias subtracted and flat-field corrected using a series of well-exposed twilight flat-fields. The absolute flux calibration was performed through observations of a series of spectrophotometric
Table 2. Spectral log.

\begin{tabular}{llll}
\hline \hline Slit centers & & Exp. times $^{a}$ & Position $^{c}$ \\
\hline$\alpha$ & $\delta$ & $(\#)^{b}$ & \\
$23^{\mathrm{h}} 49^{\mathrm{m}} 09^{\mathrm{s}}$ & $63^{\circ} 30^{\prime} 27^{\prime \prime}$ & $7800(2)$ & $\mathrm{I}$ \\
$23^{\mathrm{h}} 49^{\mathrm{m}} 18^{\mathrm{s}}$ & $63^{\circ} 33^{\prime} 06^{\prime \prime}$ & $3600(1)$ & II \\
$23^{\mathrm{h}} 55^{\mathrm{m}} 45^{\mathrm{s}}$ & $62^{\circ} 53^{\prime} 12^{\prime \prime}$ & $7200(2)$ & III \\
$23^{\mathrm{h}} 58^{\mathrm{m}} 00^{\mathrm{s}}$ & $63^{\circ} 23^{\prime} 00^{\prime \prime}$ & $7800(2)$ & IV \\
\hline $00^{\mathrm{h}} 00^{\mathrm{m}} 38^{\mathrm{s}}$ & $63^{\circ} 16^{\prime} 03^{\prime \prime}$ & $7200(2)$ & V \\
$00^{\mathrm{h}} 02^{\mathrm{m}} 55^{\mathrm{s}}$ & $63^{\circ} 17^{\prime} 59^{\prime \prime}$ & $7200(2)$ & VI \\
$00^{\mathrm{h}} 02^{\mathrm{m}} 32^{\mathrm{s}}$ & $63^{\circ} 30^{\prime} 04^{\prime \prime}$ & $7200(2)$ & VII \\
$23^{\mathrm{h}} 58^{\mathrm{m}} 22^{\mathrm{s}}$ & $63^{\circ} 55^{\prime} 14^{\prime \prime}$ & $3600(1)$ & VIII \\
\hline
\end{tabular}

${ }^{a}$ Total exposure time in $\mathrm{s}$.

${ }^{b}$ Number of spectra acquired.

${ }^{c}$ Marked as in Fig. 1.

standard stars (HR 5501, HR 7596, HR 7950, HR 9087, HR 718 and HR 8634; Hamuy et al. 1992, 1994). Flux measurements in the overlapping areas, between the August 2002 and June 2004 observations, show very good agreement (better than $10 \%)$.

\subsection{Optical spectra}

Long slit spectra in the area of $\mathrm{G} 116.5+1.1$ were obtained on July 10 and August 10, 11 and 13, 2002 with the $1.3 \mathrm{~m}$ Ritchey-Cretien telescope at Skinakas Observatory. Additional spectroscopy was performed on July 13 and 14, 2004 with the same configuration as in the earlier observations consisting of a 1300 line $\mathrm{mm}^{-1}$ grating and a $800 \times 2000$ SITe CCD covering the range of $4750 \AA-6815 \AA$. The slit width was 7 '! 7 , the slit length was 7'.9 and it was always oriented in the south-north direction. The coordinates of the slit centers, the number of available spectra and the total exposure times are given in Table 2. Sky background was always taken from the same frame as the source spectrum in areas of little or no measureable emission. Standard IRAF tasks were used to reduce the source and the auxiliary frames. Absolute flux calibration was performed through observations of the spectrophotometric standard stars HR 7596, HR 7950, and HR 9087, HR 718 and HR 8634 (Hamuy et al. 1992, 1994).

\subsection{The $\mathrm{H} \alpha+[\mathrm{NII}$ and [S II] line images}

The field shown in Fig. 1 encorporates several diffuse, patchy as well as filamentary structures. The known supernova remnant $\mathrm{G} 116.5+1.1$ is located to the lower-right where the single $1400 \mathrm{MHz}$ contour marks the actual location and size of this object, while the circle drawn to the upper-left encloses the new filamentary features that were discovered in this area. These new features may suggest the detection of a previously unknown remnant. Finally, part of the well-known supernova remnant CTB 1 can be seen in the middle-bottom area of the figure. 


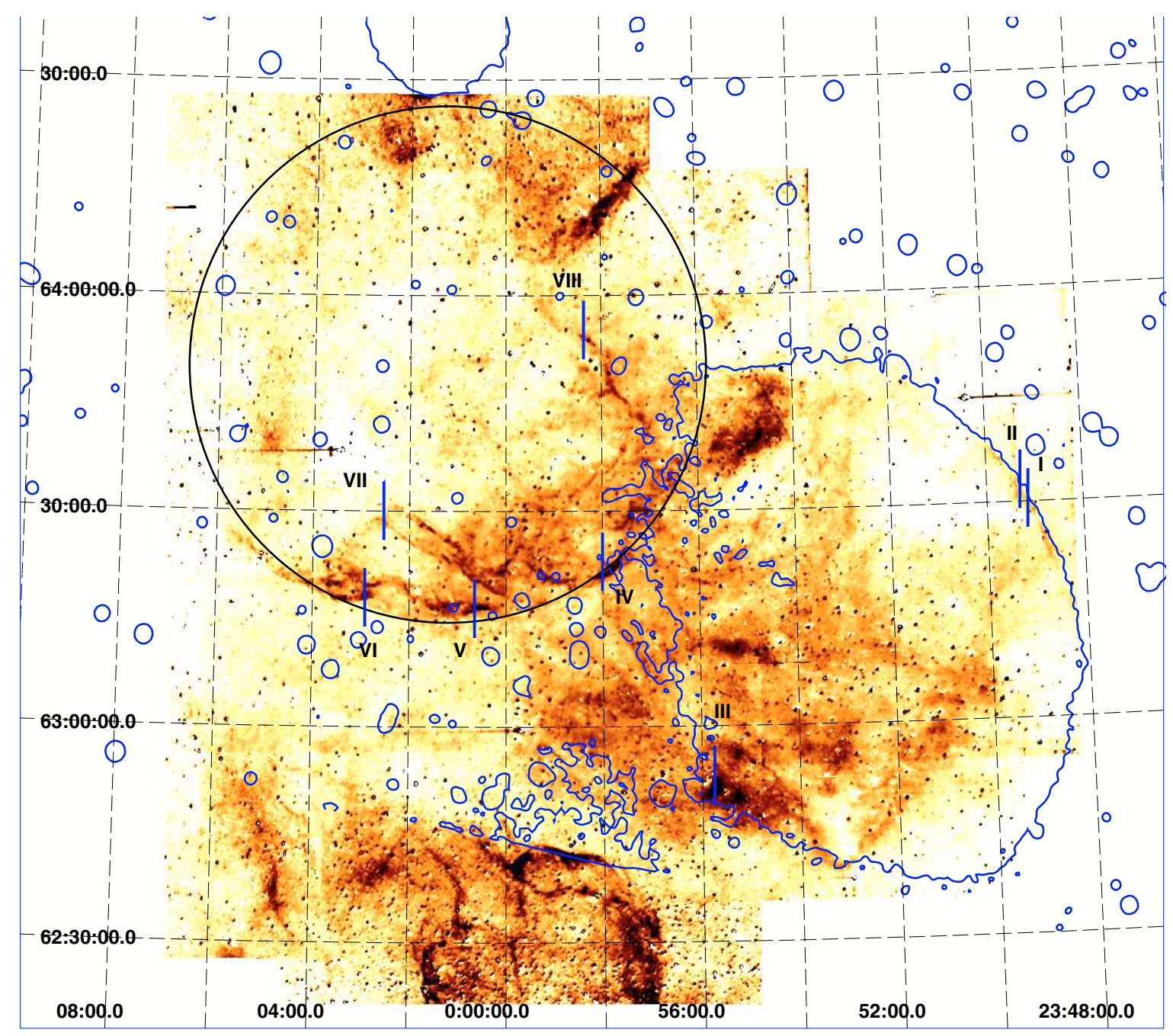

Fig. 1. Diffuse and patchy emission as well as new filamentary structures are detected in the observed fields. The known supernova remnant G 116.5+1.1 is located to the lower-right, where the $1400 \mathrm{MHz}$ contour marks the remnant's actual location and size. The circle seen to the upper-left part of the figure encompasses the new filamentary structures which may be part of a new candidate remnant. The filaments seen in the middle-bottom part of the figure constitute the upper portion of the well-known remnant CTB 1. The long rectangles mark the slit projections on the sky and are numbered from I through VIII. The line segments seen near over-exposed stars in this figure and the next figures are due to the blooming effect. The shadings run linearly from 0 to $32 \times 10^{-17} \mathrm{erg} \mathrm{s}^{-1} \mathrm{~cm}^{-2} \operatorname{arcsec}^{-2}$.

The long, faint filament detected by Fesen et al. (1997) is also present in our images and its typical intensity is a factor of 2-3 lower than the intensity of the structures found in the central to east areas of the remnant (pos. I in Fig. 1). The faint emission in the south, around a declination of $62^{\circ} 39^{\prime}$, seems to be obscured by a dust cloud giving rise to the "V" shape of the optical emission (see Sect. 5). Stronger $\mathrm{H} \alpha+[\mathrm{N} \mathrm{II}]$ emission is detected in the eastern areas of G 116.5+1.1, while the properties of the spectrum at position III (Fig. 1) may indicate emission from shock heated gas (Table 3; Sect. 4). The new imaging observations were performed, mainly in the $\mathrm{H} \alpha+$ [N II] filter, in an attempt to trace the full spatial extent of the emission to the east of G $116.5+1.1$. The diffuse emission in the very north edge of our field of view is part of LBN 117.62+02.29 (Lynds 1965), an H II nebulosity, while we were not able to find a registered entry in the SIMBAD database for the structure around $\alpha \simeq 23^{\mathrm{h}} 58^{\mathrm{m}}, \delta \simeq 64^{\circ} 10^{\prime}$, extending for $\sim 16^{\prime}$ in the south-east to north-west direction.

\section{The imaging observations}

The overall morphology in the [S II] filter (Fig. 2) is similar to that of the $\mathrm{H} \alpha+[\mathrm{N}$ II] filter, however, some structures in the field appear stronger in this line than others. This is the first indication that we may have detected emission from shock heated gas. We take advantage of our flux calibrated images (see also Mavromatakis et al. 2004, for a description of the method) to estimate roughly the $[\mathrm{S} \mathrm{II}] / \mathrm{H} \alpha$ ratio over the observed areas. The sulfur to $\mathrm{H} \alpha$ ratios are calculated only for those areas where the scatter of the measured fluxes, among the different nights of observation, is $\sim 15 \%$ or less. It is found that over the area of G $116.5+1.1$ most of the detected emission is likely to originate from photoionized gas with the exception of the known filamentary emission in the west. Emission in area III can be asssociated with the remnant but more observations are required to verify this. 
Table 3. Relative line fluxes.

\begin{tabular}{|c|c|c|c|c|c|c|c|}
\hline & & Pos. Ia & Pos. Ib & Pos. II & Pos. IIIa & Pos. IIIb & Pos. IV \\
\hline \multicolumn{2}{|l|}{ Line $(\AA)$} & $F^{a, b}$ & $F^{a, b}$ & $F^{a, b}$ & $F^{a, b}$ & $F^{a, b}$ & \\
\hline \multicolumn{2}{|l|}{$4861 \mathrm{H} \beta$} & - & - & - & $19(3)$ & $15(2)$ & $14(2)$ \\
\hline \multicolumn{2}{|l|}{4959 [O III] } & - & - & - & - & - & - \\
\hline \multicolumn{2}{|l|}{5007 [O III] } & - & - & - & - & - & - \\
\hline \multicolumn{2}{|l|}{6300 [O I $]$} & $29(3)$ & $21(5)$ & - & $5(3)$ & $8(3)$ & $14(3)$ \\
\hline \multicolumn{2}{|l|}{6364 [O I] } & - & - & - & - & - & - \\
\hline \multicolumn{2}{|l|}{6548 [N II] } & - & $16(4)$ & $7(1)$ & $9(5)$ & $6(2)$ & $7(2)$ \\
\hline \multicolumn{2}{|l|}{$6563 \mathrm{H} \alpha$} & $100(13)$ & $100(23)$ & $100(10)$ & $100(51)$ & $100(35)$ & $100(27)$ \\
\hline \multicolumn{2}{|l|}{6584 [N II] } & $43(6)$ & $50(12)$ & $40(4)$ & $41(21)$ & 45 (16) & $51(13)$ \\
\hline \multicolumn{2}{|l|}{6716 [S II] } & $47(6)$ & $52(14)$ & $36(4)$ & $22(12)$ & $27(11)$ & $50(14)$ \\
\hline \multicolumn{2}{|l|}{6731 [S II] } & $32(4)$ & $39(11)$ & $33(4)$ & $22(12)$ & $27(11)$ & $47(14)$ \\
\hline \multicolumn{2}{|c|}{ Absolute $\mathrm{H} \alpha$ flux $^{\mathrm{c}}$} & 3.8 & 5.1 & 9.1 & 14.1 & 13.9 & 11.5 \\
\hline \multicolumn{2}{|l|}{$\mathrm{H} \alpha / \mathrm{H} \beta$} & - & - & - & $5.3(3)$ & $6.6(2)$ & $6.9(2)$ \\
\hline \multicolumn{2}{|l|}{$[\mathrm{S}$ II $] / \mathrm{H} \alpha$} & $0.79(7)$ & $0.91(14)$ & $0.69(5)$ & $0.44(16)$ & $0.54(14)$ & $0.97(16)$ \\
\hline \multicolumn{2}{|c|}{$\mathrm{I}(6716) / \mathrm{I}(6731)$} & $1.47(4)$ & $1.32(8)$ & $1.08(3)$ & $1.0(8)$ & $1.0(8)$ & $1.1(10)$ \\
\hline & \multicolumn{2}{|c|}{ ( } & Pos. V & Pos. VI & Pos. VII & Pos. VIII & \\
\hline & \multicolumn{2}{|c|}{ Line $(\AA ̊)$} & $F^{a, b}$ & $F^{a, b}$ & $F^{a, b}$ & $F^{a, b}$ & \\
\hline & \multicolumn{2}{|c|}{$4861 \mathrm{H} \beta$} & $13(4)$ & $18(6)$ & $21(3)$ & $9(2)$ & \\
\hline & \multicolumn{2}{|c|}{4959 [O III] } & - & - & - & - & \\
\hline & \multicolumn{2}{|c|}{5007 [O III] } & - & $9(4)$ & - & - & \\
\hline & \multicolumn{2}{|c|}{$6300[\mathrm{OI}]$} & $17(12)$ & - & - & - & \\
\hline & \multicolumn{2}{|c|}{$6364[\mathrm{OI}]$} & $6(4)$ & - & - & - & \\
\hline & \multicolumn{2}{|c|}{6548 [N II] } & $11(8)$ & $15(12)$ & $13(2)$ & $10(2)$ & \\
\hline & \multicolumn{2}{|c|}{$6563 \mathrm{H} \alpha$} & $100(70)$ & $100(76)$ & $100(20)$ & $100(19)$ & \\
\hline & \multicolumn{2}{|c|}{6584 [N II] } & $46(32)$ & $48(37)$ & $30(6)$ & $42(8)$ & \\
\hline & \multicolumn{2}{|c|}{6716 [S II $]$} & $55(42)$ & $60(50)$ & $51(13)$ & $60(12)$ & \\
\hline & \multicolumn{2}{|c|}{6731 [S II] } & $38(30)$ & $43(35)$ & $36(9)$ & $42(8)$ & \\
\hline & \multicolumn{2}{|c|}{ Absolute $\mathrm{H} \alpha$ flux $^{\mathrm{c}}$} & 16.2 & 16.6 & 8.6 & 8.7 & \\
\hline & \multicolumn{2}{|c|}{$\mathrm{H} \alpha / \mathrm{H} \beta$} & $7.9(4)$ & $5.7(6)$ & $4.8(3)$ & $11(1)$ & \\
\hline & {$[\mathrm{S} \mathrm{II}] / \mathrm{Hc}$} & & $0.93(41)$ & $1.03(47)$ & $0.87(12)$ & $1.02(11)$ & \\
\hline & $\mathrm{I}(6716) /$ & $(6731)$ & $1.44(24)$ & $1.40(29)$ & $1.4(7)$ & $1.4(7)$ & \\
\hline
\end{tabular}

The $[\mathrm{S} \mathrm{II}] / \mathrm{H} \alpha$ ratios over the newly detected structures in the north-east strongly support the fact that the gas has been shock heated rather than photoionized. This conclusion is verified by the deep long slit spectra which offer more accurate measurements of the individual line fluxes (Sect. 4). No significant [O III] emission was detected and the $3 \sigma$ upper limit, over the area of $\mathrm{G} 116.5+1.1$ and the new fields, is $\sim 4 \times 10^{-17} \mathrm{erg} \mathrm{s}^{-1} \mathrm{~cm}^{-2} \operatorname{arcsec}^{-2}$.

\section{The long slit spectra of $G 116.5+1.1$}

Deep long slit spectra were taken in order to establish accurately the nature of the observed emission by measuring the strengths of the $\mathrm{H} \alpha$ and [S II] emission lines. A number of spectra, extracted from individual data frames, are shown in Fig. 3 and the measured fluxes are given in Table 3. 


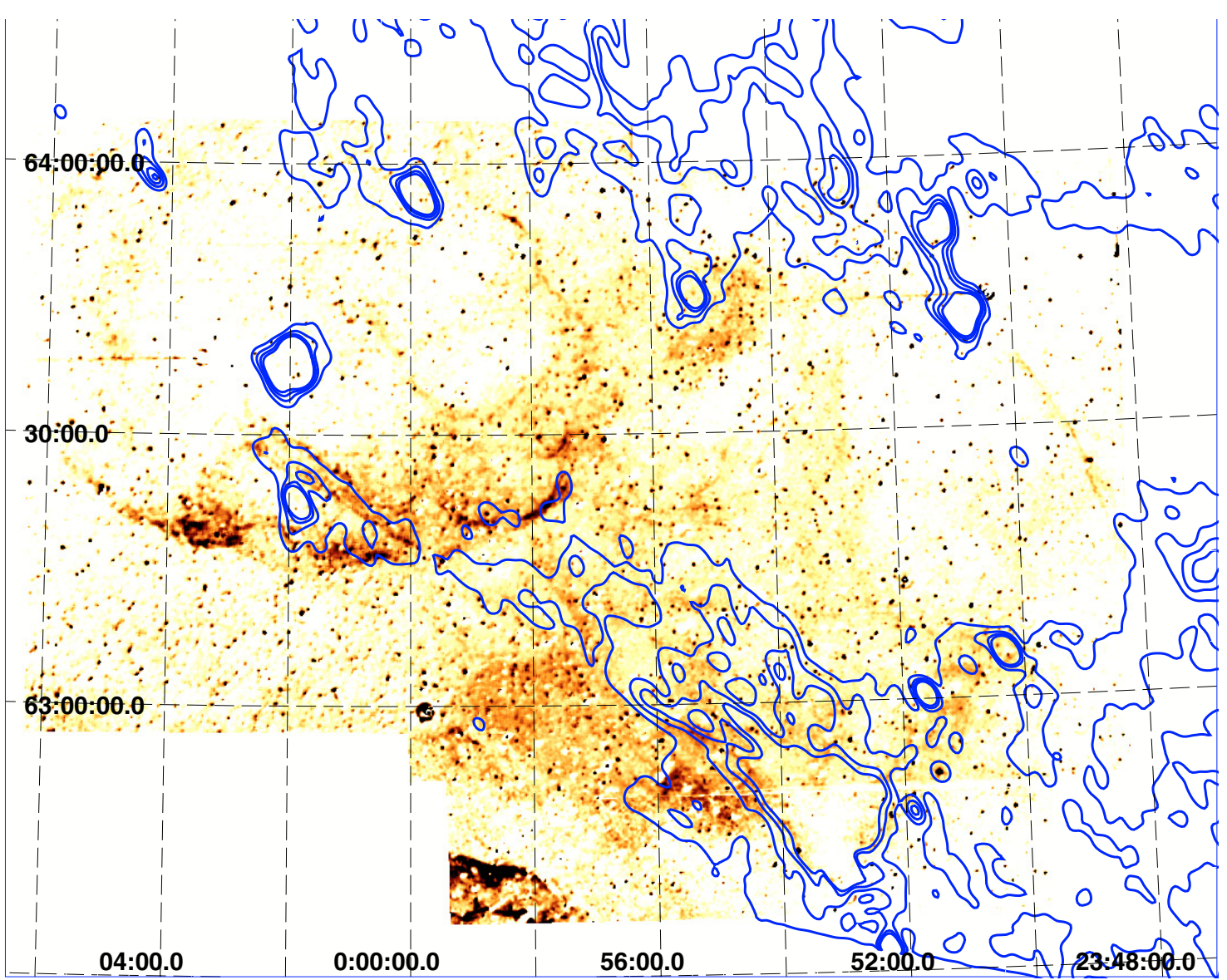

Fig. 2. The field of the remnant seen through the sulfur filter. The shadings run linearly from 0 to $9 \times 10^{-17} \mathrm{erg} \mathrm{s}^{-1} \mathrm{~cm}^{-2} \operatorname{arcsec}^{-2}$, while the $60 \mu \mathrm{m}$ contours scale linearly from 11 to $16.4 \mathrm{MJy} \mathrm{sr}^{-1}$, every $1.8 \mathrm{MJy} \mathrm{sr}^{-1}$. It may be possible that cool dust is associated with the two filaments extending to the north-east around $\alpha \simeq 00^{\mathrm{h}} 01^{\mathrm{m}} 30^{\mathrm{s}}$, and $\delta \simeq 63^{\circ} 26^{\prime}$; short segments of infrared emission also overlap optical emission around pos. IV (cf. Fig. 1). The intensity of these dust structures is typically $10 \sigma$ above the local IR background.

A set of spectra were obtained to the west of $\mathrm{G} 116.5+1.1$ (pos. I, II) and a [S II] $/ \mathrm{H} \alpha$ ratio $\sim 0.9$ was measured (Table 3), identifying the optical emission as emission from shock heated gas (see also Fesen et al. 1997). Two different apertures were extracted from the spectrum obtained at pos. III. The sulfur to $\mathrm{H} \alpha$ ratios are 0.4 and 0.5 , close to the lower limit of values for supernova remnants and this does not allow us to unambiguously identify the origin of this emission as arising from shock heated gas. The origin of the emission remains an open question, although the [S II] image (Fig. 2) shows enhanced emission in this area. We note here that the $[\mathrm{S} \mathrm{II}] / \mathrm{H} \alpha$ ratio, in known SNRs, is typically greater than 0.4 and mainly above 0.5, (e.g. Fesen et al. 1985; Raymond et al. 1988; Smith et al. 1993), while in H II regions it is found below $\sim 0.35$ and mostly around 0.2 (e.g. Hunter et al. 1992).

Five different positions were observed spectroscopically in the area of the new filaments. The properties of the spectra at positions IV, V, VI and VII strongly point to emission from shock heated gas ([S II $] / \mathrm{H} \alpha$ ratios close to 1 ; Table 3 ). The filamentary nature of the newly discovered optical radiation, as seen in the narrow band images, also supports this conclusion. Although, the low ionization lines are quite strong, [O III] line emission at $5007 \AA$ is only detected at pos. VI (Table 3 and Fig. 3) and its flux is consistent with the upper limit obtained from the imaging observations. Finally, a single spectrum was obtained in the north where another filamentary structure is detected (pos. VIII). The spectrum here suggests that the observed emission originates from shock heated gas.

In those cases where the sulfur lines are accurately measured, the implied electron densities are low, e.g. less than $180 \mathrm{~cm}^{-3}$ at the $3 \sigma$ limit at pos. VI. However, if the sulfur lines are weak and thus, of lower significance, the electron densities can cover a wide range of values, e.g. from 30 to $1400 \mathrm{~cm}^{-3}$ at the $3 \sigma$ limit at pos. IV.

In the absence of reliable $\mathrm{H} \beta$ measurements for G 116.5+1.1, we adopt the statistical relation of Predehl and Schmitt (1995) to estimate the color excess. The total galactic $N_{\mathrm{H}}$ in the direction of G $116.5+1.1$ is $7.3 \times 10^{21} \mathrm{~cm}^{-2}$, according to the FTOOLS command "nh" based on data from Dickey and Lockmann (1990). However, the velocity resolved $N_{\mathrm{H}}$ maps of Hartmann and Burton (1997) allow us to estimate a column density of $\sim 5 \times 10^{21} \mathrm{~cm}^{-2}$, assuming that the remnant is indeed in the Perseus Arm (Reich and Braunsfurth 1981). The color excess is then 1.09, equivalent to a logarithmic interstellar extinction of 1.66. Sufficient $\mathrm{H} \beta$ emission has been detected only at pos. VI, in the area of the new filamentary structures. An interstellar extinction of 0.8 is calculated $(\mathrm{H} \alpha / \mathrm{H} \beta=5.6)$, which is equivalent to a color excess of 0.5 . 


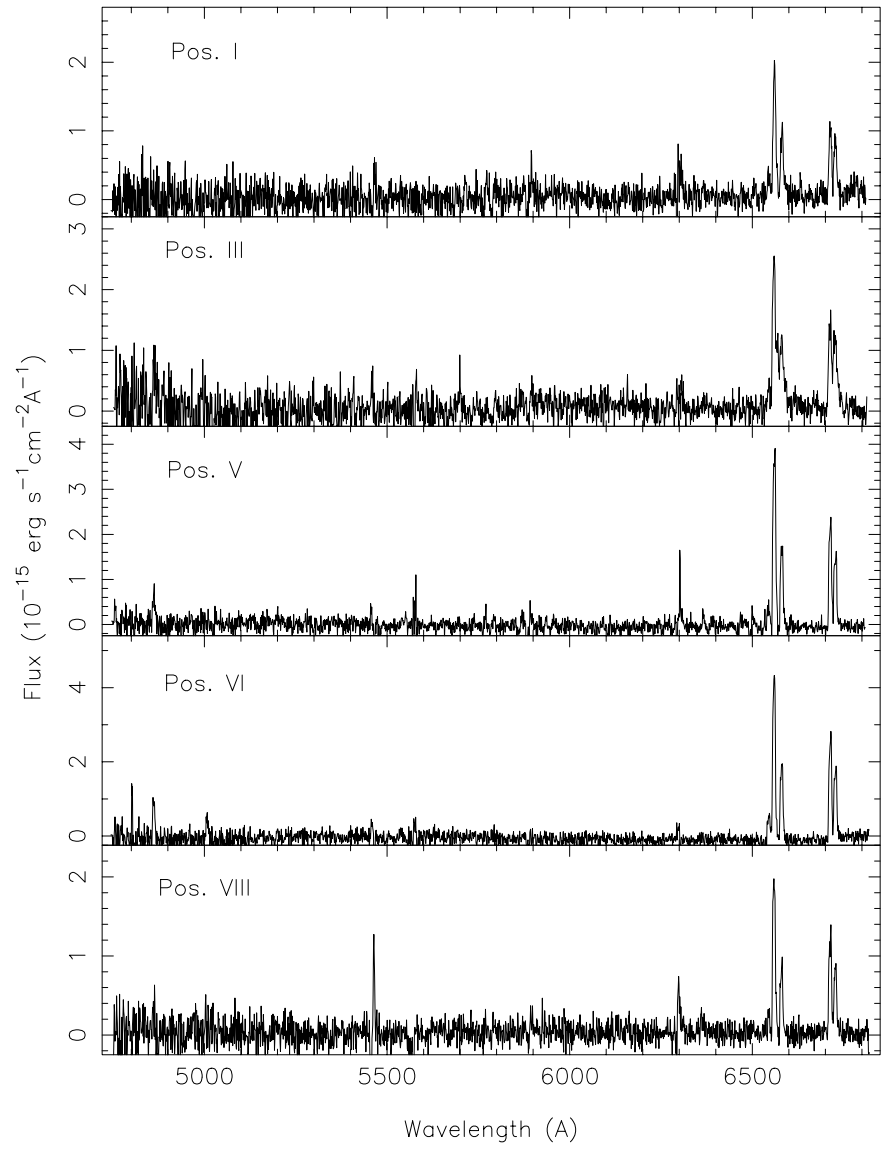

Fig. 3. Long slit spectra from individual frames extracted from the listed positions in the area of $\mathrm{G} 116.5+1.1$ and its surrounding area. More details can be found in Sect. 4 and Table 3.

\section{Observations in other wavelengths}

X-ray counterpart emission, above the sky background, has not been detected in the area of $\mathrm{G} 116.5+1.1$ or the new candidate remnant in the ROSAT All Sky survey. A possibly extended X-ray source (1RXS J235257.3+625002) is present in the south but it is not at all clear if it is related to $\mathrm{G} 116.5+1.1$. Despite the failure to detect soft X-rays, HI kinematic data (Taylor et al. 2003) as well as high resolution infrared data (Cao et al. 1997; Kerton \& Martin 2000) are available and these could provide additional information about the properties of this remnant.

New HI kinematic data are available from the Canadian Galactic Plane Survey (CGPS; Taylor et al. 2003). In Fig. 4 we have split the velocity interval from $-70 \mathrm{~km} \mathrm{~s}^{-1}$ to $-30 \mathrm{~km} \mathrm{~s}^{-1}$ into eight narrower channels and radio contours at $1400 \mathrm{MHz}$ are shown on top of the HI emission. The depression of the HI emissivity shown in Fig. 6 of Reich \& Braunsfurth (1981) is mainly present between -67.4 and $-61.0 \mathrm{~km} \mathrm{~s}^{-1}$. The $\mathrm{HI}$ images in the range of -45 to $-36 \mathrm{~km} \mathrm{~s}^{-1}$ are interesting because intense HI emission is present just outside the radio boundaries in the north and south of the remnant. Weaker and diffuse emission appears to be projected over the west part of the remnant. The HI emission is significantly reduced over the north-east part of $\mathrm{G} 116.5+1.1$ where the radio emission is also weak with respect to other areas of the remnant. If the reduced
$\mathrm{HI}$ emission at the center and the intense $\mathrm{HI}$ emission at the boundaries of $\mathrm{G} 116.5+1.1$ are due to its expanding envelope then we can estimate distances in the range of 2.5 to $3.5 \mathrm{kpc}$ (Clemens 1985). However, the actual distance depends also on the adopted galactic rotation curve even in the case where a correlation is well established.

We have also examined infrared (IR) data at $8.28 \mu \mathrm{m}$ $\left(\mathrm{MSX}^{1}\right.$ ), and 12, 25, 60 and $100 \mu \mathrm{m}$ (IRAS). In Fig. 2, the $60 \mu \mathrm{m}$ data are shown in the form of light, thin contours along with the $1400 \mathrm{MHz}$ radio emission (dark, thick contours). Intense dust emission in the south-east of $\mathrm{G} 116.5+1.1$ results in the "V" shape of the optical radiation. A close-up of this area is shown in Fig. 5, where the $8.28 \mu \mathrm{m}$ emission displays a comet-like structure. The bulk of the IR emission is confined in an area less than $\sim 10^{\prime}$ long, while the overall structure extends for $\sim 30^{\prime}$. The right plot in Fig. 5 shows the HI emission in the velocity range -45 to $-41 \mathrm{~km} \mathrm{~s}^{-1}$ along with contours of the $60 \mu \mathrm{m}$ emission. The intense IR and HI emission seem to match spatially, while there is also good positional agreement in the two lanes of emission extending to the north-east. If the correlation proposed in Fig. 5 is true, then it would imply that the optically emitting gas is further than $\sim 3 \mathrm{kpc}$ away (Clemens 1985).

Further to the north-east in Fig. 2, it can be seen that IR emission traces the two filamentary structures present between a right ascension of $00^{\mathrm{h}} 00^{\mathrm{m}}$ and $02^{\mathrm{h}} 00^{\mathrm{m}}$, just south of the declination of $63^{\circ} 30^{\prime}$. In addition, patchy IR emission at pos. IV appears to match the optical emission at this location and a correlation could be possible. The intensity of the IR emission, of $\sim 11 \mathrm{MJy} \mathrm{sr}^{-1}$, is $10 \sigma$ above the neighbouring background level of $\sim 8 \mathrm{MJy} \mathrm{sr}^{-1}$.

\section{Discussion}

The supernova remnant $\mathrm{G} 116.5+1.1$ was observed in major optical emission lines and deep long slit spectra were acquired at a few positions. The overall morphology is similar among the low ionization images (Figs. 1 and 2). New patchy and diffuse structures are detected in the area of $\mathrm{G} \mathrm{116.5+1.1} \mathrm{but}$ they do not seem related to it. There is an area of emission in the south-east which could be related to the remnant and this is the emission detected around pos. III. One of the extracted apertures is characterized by $[\mathrm{S} \mathrm{II}] / \mathrm{H} \alpha \sim 0.5$ and weak [O I] $6300 \AA$ emission, while the [S II] image shows enhanced radiation in this area compared to neighbouring positions. Its non-filamentary nature is not necessarily an indication of photoionized gas since remnants, like e.g. W44, display similar morphologies (Raymond \& Curiel 1995; Mavromatakis et al. 2003). The lower $[\mathrm{S} \mathrm{II}] / \mathrm{H} \alpha$ ratio measured here, compared to the north-west, could be explained by a lower ionization state of the preshocked gas and/or by a stronger than usually assumed magnetic field (e.g. Raymond 1979; Cox \& Raymond 1988).

The long slit spectra from the area of G $116.5+1.1$ set an even lower upper limit to any [O III] emission at the specified

1 The MSX data were retrieved from the NASA/IPAC Infrared science archive at http://irsa.ipac. caltech.edu 

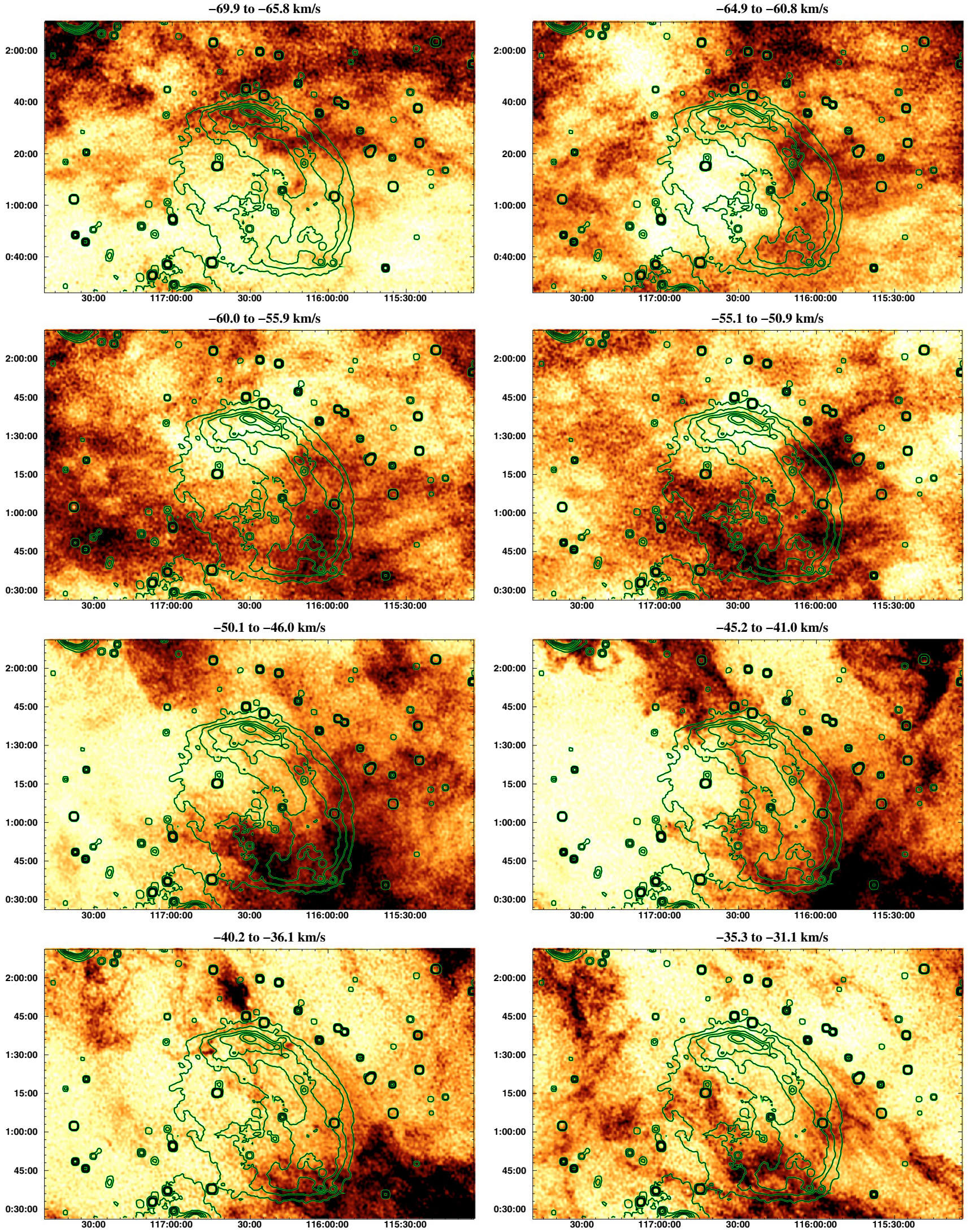

Fig. 4. The HI emission in the range of LSR velocities -70 to $-31 \mathrm{~km} \mathrm{~s}^{-1}$ has been split into eight narrower velocity intervals. The radio contours at $1400 \mathrm{MHz}$ scale, every $0.22 \mathrm{~K}$, from 5.7 to $6.8 \mathrm{~K}$ brightness temperature. The data are plotted in galactic coordinates and more details are given in Sect. 5. 

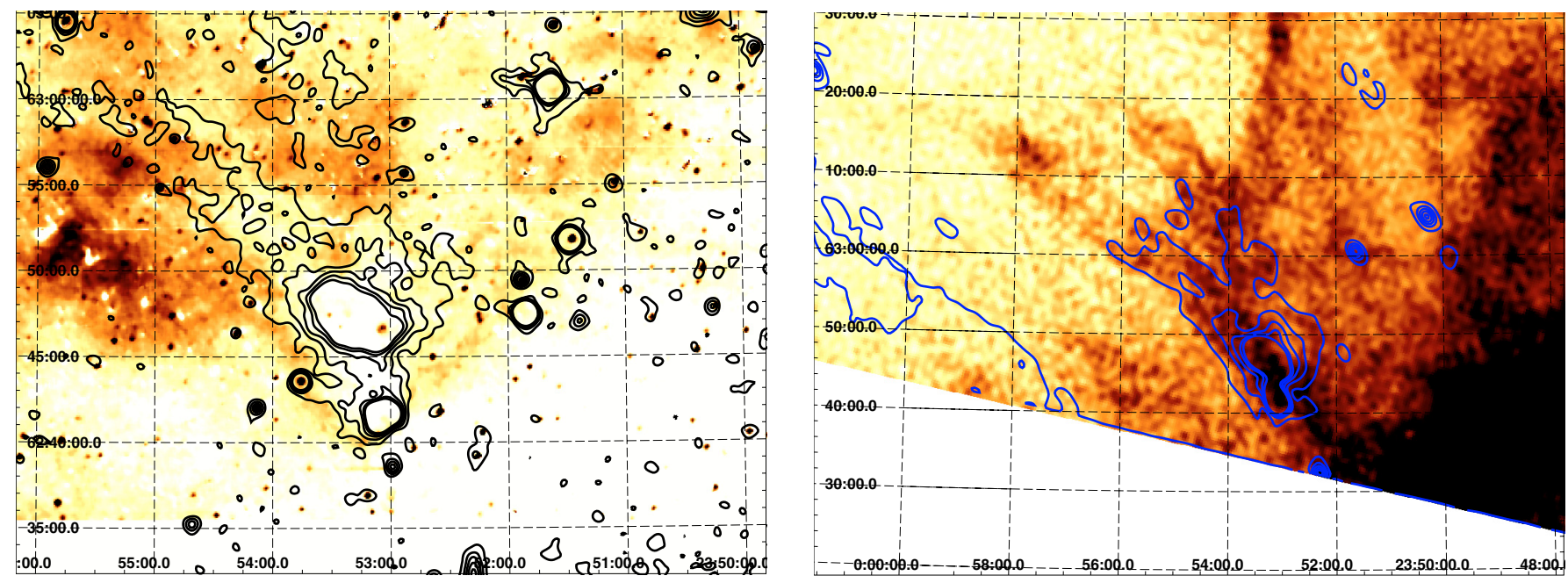

Fig. 5. The left figure shows the optical emission in the south of G 116.5+1.1 along with high resolution MSX data at $8.28 \mu \mathrm{m}$. The IR emission is nicely anticorrelated with the $\mathrm{H} \alpha+[\mathrm{N}$ II] emission. Note that the central IR emission is a factor of $\sim 15$ stronger than the emission of the tail extending to the north-east. The right figure shows the $\mathrm{HI}$ emission in a wider area and in the range of LSR velocities -45 to $-41 \mathrm{~km} \mathrm{~s}^{-1}$, while the overlaid contours outline the $60 \mu \mathrm{m}$ IR data $\left(1^{\prime} \times 1\right.$ 1.7 resolution; Cao et al. 1997) and is seen that the infrared emission also extends to the north-east.

positions compared to the imagery. The [O III] flux production depends mainly upon the shock velocity and the ionization state of the preshocked gas. Given the data in hand, we cannot determine whether slow shocks travel into ionized gas or whether faster shocks travel into neutral gas (Cox \& Raymond 1985), but we can exclude moderate or fast shocks overtaking ionized gas. It is evident that the [O III] image data help us to generalize this conclusion and apply it to the whole area of the remnant.

If the radio and $\mathrm{HI}$ emission over $\mathrm{G} 116.5+1.1$ are indeed associated in the range of -45 to $-36 \mathrm{~km} \mathrm{~s}^{-1}$, then the distance would be in the range of 2.5 to $3.5 \mathrm{kpc}$ (Sect. 5; Fig. 4). This distance is compatible with that of $3.1 \mathrm{kpc}$ calculated by Huang $\&$ Thaddeus (1986) using the $\Sigma-D$ relation, while the longer distance given by Reich and Braunsfurth (1981) may be due to the different galactic rotation curve used and the wider range of the adopted HI velocities of -60 to $-45 \mathrm{~km} \mathrm{~s}^{-1}$. Radio emission at a level of $5.8 \mathrm{MJy} \mathrm{sr}^{-1}$ best matches the filamentary optical emission in the north-west, resulting in an angular size of $79^{\prime} \times 51^{\prime}$. This is equivalent to a linear size of $69 \mathrm{pc} \times 45 \mathrm{pc}$, at a distance of $3 \mathrm{kpc}$, placing this object among the largest known supernova remnants. G $116.5+1.1$ is an interesting remnant to study, especially in X-rays, since its typical length scale is difficult to accomodate with ages of a few $10^{4}$ years and $E 51 / n_{\mathrm{o}} \sim 10$ or less (E51 stands for the explosion energy in units of $10^{51} \mathrm{erg}$ and $n_{\mathrm{o}}$ is the initial density of the ambient medium).

Several filaments have been discovered to the north-east of $\mathrm{G} 116.5+1.1$. A number of these filamentary structures (e.g. pos. IV, V, VI) trace well the periphery of the $36^{\prime}$ radius sphere whose boundary is drawn in Fig. 1. Other structures appear to be projected in the inner areas (e.g. pos. VII, VIII). Estimates of the $[\mathrm{S} \mathrm{II}] / \mathrm{H} \alpha$ ratio based on the images show that we are dealing with radiation coming from gas that has been shocked rather than photoionized. Indeed, the long slit spectra at five different positions clearly identify the emission as resulting from shock heated gas. In order to obtain estimates of the preshock cloud density $n_{\mathrm{c}}$, we assume a shock velocity in the range of 70 to $120 \mathrm{~km} \mathrm{~s}^{-1}$, given the apparent absence of [O III], and find values of $n_{\mathrm{c}}$ around $5 \mathrm{~cm}^{-3}$ or less (Fesen \& Kirshner 1980). The $\mathrm{H} \beta$ flux, although weak, is present in the majority of the spectra and this could imply that the new structures are closer to us than G 116.5+1.1. Their morphology, spatial distribution on the sky and spectral signatures lead us to propose that we have observed a new candidate remnant which we designate as $\mathrm{G} 117.4+1.5$. Furthermore, these data (optical and radio) do not support a relation between this new candidate remnant and the known remnant G 116.5+1.1.

In the area of the new candidate remnant, weak infrared emission at $60 \mu \mathrm{m}$ and $100 \mu \mathrm{m}$ appears to match the optical emission around pos. IV and VII (Fig. 2), while at shorter IR wavelengths significant emission is not detected. The CGPS data at 408 and $1400 \mathrm{MHz}$ were examined for radio emission in the area of the new candidate remnant, but we were not able to identify any morphological features that would imply a correlation with the optical emission. The failure to detect radio emission at these frequencies may lie in a number of reasons, like the magnetic field strength, the ambient medium density, shock conditions etc. (e.g. Blandford \& Cowie 1982; Pineault et al. 1997). However, this object may not be the only one where optical emission is detected but at the same time its radio emission is very weak or below detection. A candidate remnant in Cygnus, near CTB 80, displays a similar morphology in the optical and very weak radio emission (Mavromatakis \& Strom 2002). Another, very extended and evolved, candidate remnant in Pegasus shows highly filamentary structures in the optical, while associated radio emission has not been detected (Boumis et al. 2002). It may be possible that the candidate remnant $\mathrm{G} 117.4+1.5$ belongs to the same class of objects. It is 
clearly interesting to note that Blandford \& Cowie (1982) had predicted the existence of "radio quiet" remnants. New observations of this region, in wavelengths other than optical and especially in radio, would be required to validate our suggestion. In addition these observations could determine the properties of such objects and explain why they have escaped detection by current radio surveys.

\section{Conclusions}

The emission in the field of G $116.5+1.1$ is diffuse and patchy and the majority of the emission may not be related to the remnant. There is only one area in the central-east where the detected emission could be related to the remnant. If true, it would suggest that $\mathrm{G} 116.5+1.1$ is more extended in the optical regime than previously realized. The distance of $\sim 3 \mathrm{kpc}$ derived from HI kinematic data agrees fairly well with previous estimates and implies a very extended remnant.

The low ionization images and the long slit spectra of an area to the north-east of $\mathrm{G} 116.5+1.1$ show that the discovered emission most likely originates from shock heated gas. The morphology of the emission is filamentary, while some of the filaments appear aligned along the periphery of a circle of $36^{\prime}$ radius. These filaments are probably part of a previously unrecognized remnant designated as G 117.4+1.5. It is characterized by very low radio emission, below the detection limit of current radio surveys.

Acknowledgements. The authors would like to thank the referee for stimulating suggestions and comments which helped to improve and clarify the scope of this work. Skinakas Observatory is a collaborative project of the University of Crete, the Foundation for Research and Technology-Hellas and the Max-Planck-Institut für Extraterrestrische Physik. This research made use of data products from the Midcourse Space Experiment. Processing of the data was funded by the Ballistic Missile Defense Organization with additional support from the NASA Office of Space Science. The data from the Canadian Galactic Plane Survey were obtained from the Canadian Astronomy Data Centre (where the author is a guest user) which is operated by the Herzberg Institute of Astrophysics of the National Research Council Canada.

\section{References}

Blandford, R. D., \& Cowie, L. L. 1982, ApJ, 260, 625

Boumis, P., Mavromatakis, F., Paleologou, E. V., \& Becker, W. 2002, A\&A, 396, 225

Cioffi, D. F., McKee, C. F., \& Bertschinger, E. 1988, ApJ, 334, 252

Cao, Y., Terebey, S., Prince, T. A., \& Beichman, C. 1997, ApJS, 111, 387

Clemens, D. P. 1985, ApJ, 295, 422

Cox, D. P., \& Raymond, J. C. 1985, ApJ, 298, 651

Dickey, J. M., \& Lockman, F. J. 1990, ARA\&A, 28, 215

Fesen, R. A., \& Kirshner, R. P. 1980, ApJ, 242, 1023

Fesen, R. A., Blair, W. P., \& Kirshner, R. P. 1985, ApJ, 292, 29

Fesen, R. A., Winkler, F., Rathore, Y., Downes, R. A., \& Wallace, D. 1997, AJ, 113, 767

Fich, M. 1986, ApJ, 303, 465

Hamuy, M., Walker, A. R., Suntzeff, N. B., et al. 1992, PASP, 104, 533

Hamuy, M., Suntzeff, N. B., Heathcote, S. R., et al. 1994, PASP, 106, 566

Hartmann, D., \& Burton, W. B. 1997, Atlas of Galactic Neutral Hydrogen (Cambridge University Press)

Huang, Y. L., \& Thaddeus, P. 1986, ApJ, 309, 804

Hunter, D. A. 1992, ApJS, 79, 469

Kerton, C. R., \& Martin, P. G. 2000, ApJS, 126, 85

Lasker, B. M., Russel, J. N., \& Jenkner, H. 1999, in the HST Guide Star Catalog, version 1.1-ACT, The Association of Universities for Research in Astronomy, Inc.

Lynds, B. T. 1965, ApJS, 12, 163

Matthews, B. C., Wallace, B. J., \& Taylor, A. R. 1998, ApJ, 493, 312

Mavromatakis, F., \& Strom, R. G. 2002, A\&A, 382, 291

Mavromatakis, F., Boumis, P., \& Coudis, C. 2003, A\&A, 405, 591

Mavromatakis, F., Xilouris, E., \& Boumis, P. 2004, A\&A, 426, 567

Pineault, S., Landecker, T. L., \& Reich, W. 1997, A\&A, 324, 1152

Predehl, P., \& Schmitt, J. H. M. M. 1995, A\&A, 293, 889

Raymond, J. C. 1979, ApJS, 39, 1

Raymond, J. C., Hester, J. J., Cox, D., et al. 1988, ApJ, 324, 869

Raymond, J. C., \& Curiel, S. 1995, The Fifth Mexico-Texas Conference on Astrophysics: Gaseous Nebulae and Star Formation, 3, 199

Reich, W., \& Braunsfurth, E. 1981, A\&A, 99, 17

Saken, J. M., Fesen, R. A., \& Shull, J. M. 1992, ApJS, 81, 715

Smith, R. C., Kirshner, R. P., Blair, W. P., Long, K. S., \& Winkler, P. F. 1993, ApJ, 407, 564

Taylor, A. R., Gibson, S. J., Peracaula, M., et al. 2003, AJ, 125, 3145 